\title{
Intense EM Filamentation in relativistic hot plasmas
}

\author{
Qiang-Lin $\mathrm{Hu},{ }^{1}$ Zhong-Ping Chen, ${ }^{2}$ and Swadesh M. Mahajan ${ }^{2,3}$ \\ ${ }^{1}$ Department of Physics, Jinggangshan University, \\ Ji'an, Jiangxi 343009, People's Republic of China \\ ${ }^{2}$ Department of Physics and Institute for Fusion Studies, \\ The University of Texas at Austin, Austin, TX 78712, USA \\ ${ }^{3}$ Department of Physics, School of Natural Sciences, \\ Shiv Nadar University, Uttar Pradesh 201314, India
}

\begin{abstract}
Through 2D particle-in-cell (PIC) simulations, we demonstrate that the nature of filamentation of a high intensity electromagnetic (EM) pulse propagating in an underdense plasma, is profoundly affected at relativistically high temperatures. The "relativistic" filaments are sharper, are dramatically extended (along the direction of propagation), and live much longer than their lower temperature counterparts. The thermally boosted electron inertia is invoked to understand this very interesting and powerful phenomenon.
\end{abstract}

\section{Introduction}

Laser filamentation is one of the rather profound expressions of nonlinear processes unleashed during the interaction of a high amplitude laser pulse with a plasma (laser created or otherwise). The phenomenon has been vigorously investigated, both experimentally and theoretically [1-6] because of its intrinsic scientific challenges, and because of its immense relevance to astrophysics $[7,8]$ and other areas of physics [9-11].

The basic physics of filamentation, originating in the density dependence of the refractive/dispersive properties of the plasma, is quite simple. When a propagating intense laser beam encounters a density perturbation in the plasma, it is, preferentially, refracted to lower density regions (more transparent to the electromagnetic wave) boosting up the local energy density. The ponderomotive force resulting from the differential (inhomogeneous) local electromagnetic (EM) energy density, by pushing the plasma particles from the low to the high density regions, sets in the positive feed back instability that piles up more and more electromagnetic energy into the density troughs. Eventually, the laser beam filaments and begins to flows in narrow plasma channels that are characterized by high EM intensity simultaneous with low plasma density [12].

It is natural to expect that the basic laser/plasma parameters (laser power, frequency, energy distribution, plasma density and temperature $[12,13])$, that determine the linear and nonlinear refraction coefficient and the ponderomotive force, will also control the dynamics of filamentation. There are several physical observables that may be invoked to characterize filamentation: the onset time, the extension of the filament along the direction of propagation (to be called the filament length, $L_{F}$ ), and the persistence time before the filaments begin to diffuse by new nonlinear processes. Both the spatial extension and longevity of the filaments can serve as characteristic measures of the filament "quality". The primary determinants of $L_{F}$, for example, must be the basic laser/plasma parameters. It is found, however, that additional factors, like the wave polarization might also influence the filaments length; The temperature $\mathrm{T}$ and the effective mass, measured in energy units, tends to be smaller for linear polarization as compared to circular polarization due to a higher nonlinear coefficient [14,15].

Although we did list plasma temperature as a possible parameter that influences the filamentation dynamics, there is very little theoretical or experimental effort to chart out what the variation of temperature actually does. The goal of this paper, therefore, is to investigate the temperature dependence of filamentation dynamics through Particle-in-cell (PIC) simulations of the fate of a high intensity EM pulse propagating in a hot under dense plasma. Bulk relativistic electrons and ions with temperatures of multi-MeV have been achieved in laboratory $[16,17]$ and observed in astrophysical systems [18]. Multiple $\mathrm{MeV}$ plasma will be routinely created in the class of experiment called "laboratory astrophysics"[19]. Thus in our simulations, the plasma is allowed to be fully relativistic both kinematically and thermally, i.e, $T / m$ can be greater than 1 . In this paper, the temperature $T$ is measured in energy units, $m$ is the electron rest mass, and the speed of light, $c=1$.

We find that, although the filamentation dynamics is relatively insensitive to temperature variation in the nonrelativistic regime $(T \ll m \approx 500 \mathrm{keV})$, it is profoundly affected in the regime of relativistic temperatures. As $T / m$ becomes bigger than unity, the onset of filamentation is delayed, but once formed, the filaments are sharp, are spectacularly extended (larger $L_{F}$ ), and persist for a longer time. Later, we will proffer a theoretical explanation for the simulation results that we now describe.

\section{Simulation - results}

To elicit the essential features of EM filamentation in high temperature plasmas, we carried out $2 \mathrm{D}$ simulations using a fully relativistic PIC code (EPOCH) [21,22]. We study a neutral proton-electron plasma and a simulation box of size $60 \mu \mathrm{m} \times 30 \mu \mathrm{m}$ containing $900 \times 450$ cells. Initially, the ions and electrons have the designated temperature and a number density $n=n_{c} / 2\left(n_{c}\right.$ is the critical 
density) or 8000 particles per cell. A laser, linearly polarized in the $z$-direction, propagating in the $x$-direction, with intensity

$$
I=I_{0}\left[1+\delta \cos \left(k_{y} y\right)\right] \cos ^{2}\left(k_{x} x-\omega t\right)
$$

is normally incident from the left $(x=0 \mu \mathrm{m})$. In our simulations, the main part of the beam has an intensity $I_{0}=1.24 \times 10^{23} \mathrm{~W} / \mathrm{m}^{2}$ and its wavelength $\lambda=1.06 \mu \mathrm{m}$. The perturbation $\delta \cos \left(k_{y} y\right)$ has an amplitude $\delta=0.02$, and a wave vector $k_{y}=8 \pi / 30 \mu \mathrm{m}^{-1}$ in the $y$-direction. Simulations invoke periodic boundary conditions in the $y$-direction.

The most important of our results are displayed in a set of snapshots shown in Fig. 1, showing, for different initial temperatures, the filamented structure of the laser beam at three different times: $t=75,150$ and $250 \mathrm{fs}$ (evolving from an initial $(\mathrm{t}=0)$ y profile that is essentially uniform with a small $\delta=0.02$ modulation). We note:

1) For higher temperatures, the filamentation begins at later times; in the $75 \mathrm{fs}$ row, for example, the filamentation is clearly manifest at 100 and $500 \mathrm{keV}$, it is barely discernible at $1 \mathrm{MeV}$, is about to begin at $2 \mathrm{MeV}$, and is absent at $3 \mathrm{MeV}$. At a later time, $t=150 \mathrm{fs}$, the filaments are beginning to diffuse for lower temperatures, while sharp, well-formed distinctive filamented structures emerge at higher $T$; the trend continues in the $250 \mathrm{fs}$ frames; filamentation is sharpest at $3 \mathrm{MeV}$.

2) Though the filamentation has a delayed onset at high temperatures, it is at relativistic temperatures where the filamentation dynamics is most spectacular; the spatial extension $L_{F}$ of the stable filaments increases rapidly with temperature as is reflected clearly in the frames displayed in the last row of Fig. 1. From the $L_{F}$ versus $T$ curve in Fig. 2, we deduce; i) in the entire non relativistic range (unto $\mathrm{T} \approx 300 \mathrm{keV}$ ), $L_{F}$ is essentially unchanged, ii) As we enter and go deeper into the relativistic regime, the filaments live longer and become very extended in the direction of propagation; in, fact, $L_{F}$ shows an "explosive" increase after $T / m$ crosses unity.

3) In all cases, the peak electric field strength in the established filamentation is about 3 times the incident wave amplitude $\left(9.66 \times 10^{12} \mathrm{~V} / \mathrm{m}\right)$.

4) The filamentation dynamics is rather complex and multiply structured; in addition to the main filaments (large concentration of EM energy) formed about the crests of the $\delta$ perturbation, second order filaments, with somewhat weaker energy concentration, are seen to grow in between the main filaments (Fig. 1). Although it is the very sharp main filaments that represent the principle nonlinear reorganization of the plasma- laser system (instead of the leaser beam propagating with a uniform y profile, it flows through low density plasma channels), the nonlinear physics of the formation (and then weakening) of the secondary filaments is highly interesting and very involved.
5) To demonstrate that the basic characteristics of the filamentation phenomena are independent of the magnitude of the perturbation, we carried out simulations with several values of $\delta(0.01,0.02$ and 0.04$)$ and found no qualitative difference. The temperature dependence of the filamentation length, $L_{F}$ (one of the key results), was nearly the same for all values of $\delta$.

\section{Theoretical explanation - discussion}

In order to understand and properly interpret the remarkable change in dynamics brought about by relativistic temperatures, let us review, in some detail, the basic determinants of the nonlinear processes that lead to filamentaion.

It was mentioned earlier that the density dependent refractive properties of the plasma, in conjunction with the ponderomotive force, may be the root cause of filament formation. That statement, however, is only partially correct. Although for non-relativistic temperatures, the refractive properties do, indeed, remain essentially independent of thermal energy, they are strongly affected in the regime of relativistic temperatures. Let us examine the dispersion relation of an EM wave of arbitrary amplitude $q A / m$, where $q$ is the particle charge, and $A$ is the magnitude of the vector potential [15]

$$
\omega^{2}-k^{2}=\frac{\omega_{p}^{2}}{\Gamma_{f} \Gamma_{t h}}
$$

where $\omega_{p}=\sqrt{4 \pi q^{2} n_{R} / m}$ is the invariant (rest frame) plasma frequency and $n_{R}$ is the rest frame density. Of the two $\Gamma$ factors,

$$
\begin{gathered}
\Gamma_{f}=\sqrt{\left(1+\frac{q^{2} A^{2}}{m^{2}}\right)}, \\
\Gamma_{t h}=K_{2}(\zeta) / K_{1}(\zeta), \zeta=\frac{\sqrt{\left(1+q^{2} A^{2} / m^{2}\right)}}{T / m}
\end{gathered}
$$

that distinguish Eq.(??) from its simplest non relativistic form, $\Gamma_{f}$, the field- enhanced mass factor (loosely called the relativistic mass increase) is well-known, while the displayed expression for $\Gamma_{t h}$ is the result of a very recent calculation where a statistical description of weakly interacting quantum particles subject to an arbitrary strength EM field is constructed [16]. In Eq.(??), $K_{n}(\zeta)$ are the modified Bessel functions of order $n$.

The dispersion relation (??) tells us that in addition to the rest frame density $n_{R}$, two additional parametersthe strength of the EM wave $(q A / m)$ and the normalized temperature $(T / m)$ - are essential determinants of the propagation characteristics (cutoff frequency, critical density etc.) of a relativistically intense EM wave in a hot plasma. The temperature factor $\Gamma_{t h}>1$ is the fundamental reason why the filamentation process becomes more pronounced as one goes to relativistic temperatures 
because it is then that $K_{2}(\zeta) / K_{1}(\zeta)$ can become considerably greater than unity (For $\left.T / m \ll 1, \Gamma_{t h} \approx 1\right)$

The role of $\Gamma_{t h}>1$ could be assessed in several equivalent ways- it is naturally interpreted as the increase in effective mass due to thermal energy just as $\Gamma_{f}$ reflects the boost in effective mass due to the EM energy. Both of these processes, by lowering the cutoff frequency, enhance transparency to the EM waves.

Let us now construct a qualitative picture of how the filamentaion dynamics is so strongly affected for $T / m>1$. When the laser beam enters the plasma, the ponderomotive force caused by the $\delta$ inhomogeneity in intensity, pushes the electrons out of the higher intensity region lowering the local electron density. And since the lower density regions, because of their greater transparency, are more receptive to the EM wave, the local EM intensity is further boosted. The feedback process continues till EM energy tends to flow in narrow filaments around the peaks of the initial beam. Since the plasma transparency (for a given initial density $n_{R}$ ) is considerably higher at relativistic temperatures as compared to the non-relativistic ones, the nonlinear processes that cause filamentation become commensurately stronger resulting in sharp, extended and long lived plasma channels through which the EM energy flows.

Naturally, the regions of high EM energy (the laser filaments) are also the regions of lower electron density as shown in Fig. 3, where we have displayed only the highest temperature $(2$ and $3 \mathrm{MeV})$ cases. The final form for the simultaneous channel-filament structure is determined by a complex interaction of the ponderomotive, thermal pressure, and the charge separation forces; the emergence of the latter speeds up the movement of sluggish ions while slowing down the more mobile electrons. The details will be given in a more comprehensive paper.

Although the filament "quality" and duration are considerably boosted at high temperatures, the filaments will eventually decay due to the development of various instabilities like the stimulated Raman scattering.

In order to check the essentials of the proffered explanation, that the thermally enhanced electron effective mass $\left(m \Gamma_{t h}\right)$ may be the cause of the interesting and profound change in the dynamics of filamentation, we conducted a set of simulations with "electrons" that are thermally non-relativistic but whose rest mass is artificially jacked. We display in Fig. 4 the simulation results for $m^{*}=6 m$ and $T=100 \mathrm{keV}$ keeping all other conditions to be the same as before.

The comparison of Fig. 4 and the snapshots in the first column of Fig.1(corresponding to $T=100 \mathrm{keV}$ ) show, as expected, that the filaments are formed later, are sharper, and spatially extended at the artificially assigned larger mass; in Fig. 4, distinct filaments emerge after $t=300 \mathrm{fs}$, and their length is about $44 \mu \mathrm{m}$ at $t=400 \mathrm{fs}$. Thus for both cases, where the electron effective mass was enhanced to relativistic values, whether thermally $(T=3 \mathrm{MeV}$, rest mass $m)$ or artificially $\left(T=100 \mathrm{keV}, m^{*}=6 m\right)$, the filamentation dynamics is profoundly effected in qualitatively similar ways. One does not expect an exact equivalence because the thermally enhanced mass factor is a bit more complicated than a simple proportionality (as can be seen from the $\Gamma$ factors). The detailed comparison will require more comprehensive work in which the intertwining EM and thermal effects are treated, more accurately, for the configuration relevant to the presented simulations [20].

Although not central to the main theme of this letter, one notes with great interest the emergence, and then dissipation, of the second order filaments (second order plasma channels) around the troughs of the $\delta$ perturbation. The fascinating nonlinear physics, responsible for this phenomenon, though straightforward, is sufficiently complex to merit a separate paper. Explanation of the second order order filaments, along with a more quantitative investigation of the filamentation process (see the preceding paragraph) will be presented in a forthcoming paper.

The PIC simulations of a high intensity laser wave traveling in an arbitrary temperature under-dense plasma show that the process of EM filamentation (EM energy flowing through narrow plasma channels) undergoes a spectacular change at relativistic temperatures; the (relativistic) filaments become very sharp and extended in the direction of propagation, and persist for much longer times. The nonlinear processes that lead to filamentation become more effective due to the thermally enhanced plasma transparency. Here is, then, a very effective physical mechanism that can be exploited to achieve an even greater spatial concentration of EM energy.

References

[1] N. B. Meezan, L. Divol, M. M. Marinak, et al., Phys. Plasmas 11, 5573 (2004).

[2] E. M. Epperlein, Phys. Rev. Lett. 65, 2145 (1990).

[3] A. J. Schmitt, Phys. Fluids 31, 3079 (1988).

[4] P. E. Young, Phys. Plasmas 2, 2825 (1995).

[5] S. Wilks, P. E. Young, J. Hammer, et al., Phys. Rev. Lett. 73, 2994 (1994).

[6] G. Sarri, C. A. Cecchetti, R.Jung, et al., Phys. Rev. Lett. 106, 095001 (2011).

[7] A.L. Peratt, Astrophysics \& Space Science 227, 97(1995).

[8] C. Ruyer, L. Gremillet, A. Debayle, et al., Phys. Plasmas 22, 032102 (2015).

[9] Hai-Wei Du, H. Hoshina, C. Otani, K. Midorikawa, Appl. Phys. Letts. 107, 211113 (2015).

[10] E. Schubert, D. Mongin, J. Kasparian,et al., Optics Express 23, 28640 (2015).

[11] H. L. Xu, P. T. Simard, Y. Kamali,et.al., Laser Physics 22, 1767 (2012).

[12] V.P. Kandidov, S.A. Shlenov, O.G. Kosareva, Quantum Electronics 39, 205 (2009). 
[13] P. A. Zhokhov and A. M. Zheltikov, Phys. Rev. A 89,043816 (2014).

[14] S.Petit, A.Talebpour, A.Proulx, Chin S. Opt. Commun., 175, 323 (2000).

[15] Swadesh M. Mahajan and Felipe A. Asenjo, Physics of Plasmas 23, 056301 (2016).

[16] T. Kluge et al. Phys. Rev. Letters 107, 205003 (2011) and the reference therein.

[17] B. M. Hegelich et al., New J. Phys. 15085015 (2013).

[18] K. Hirata et al. Phys. Rev. Lett. 58, 1490 (1987).

[19] See for example the proposal/description in LLNL https://lasers.llnl.gov/science/understandingthe-universe/lab-astrophysics and Osaka University https://www.phys.sci.osakau.ac.jp/en/researchgroups/group/13-

1takabe/takabe/index.html

[20] The dispersion formula ?? was derived for a circularly polarized wave. The equivalent relation for the linearly polarized wave is considerable more complicated and is being worked on.
[21] Extendable PIC Open ration (EPOCH), [Online]. http://ccpforge.cse.rl.ac.uk/gf/project/epoch/.

[22] T. D. Arber, K. Bennett, C. S. Brady, A. Lawrence-Douglas, M. G. Ramsay, N. J. Sircombe, P. Gillies, R. G. Evans, H. Schmitz, A. R. Bell, and C. P. Ridgers, Plasma Phys. Controlled Fusion 57 (2015) 113001.

We are grateful to Dr. David Stark for helping us at all stages of the work. Simulations were performed using EPOCH code (developed under UK EPSRC grants $\mathrm{EP} / \mathrm{G} 054940 / 1$, EP/G055165/1 and EP/G056803/1) using HPC resources provided by the TACC at the University of Texas. This work was supported by US DOE Contract No. DE-FG02-04ER54742, NNSA Contract No. DE-FC52-08NA28512, DOE SCGF administered by ORISE-ORAU under Contract No. DEAC05-06OR23100, the National Natural Science Foundation of China under Grant No. 11064005, Natural Science Foundation of Jiangxi province under Grant No. 2010GQW0030, and the financial support provided by the Key Subject of Atomic and Molecular Physics in Jiangxi Province (2011-1015).
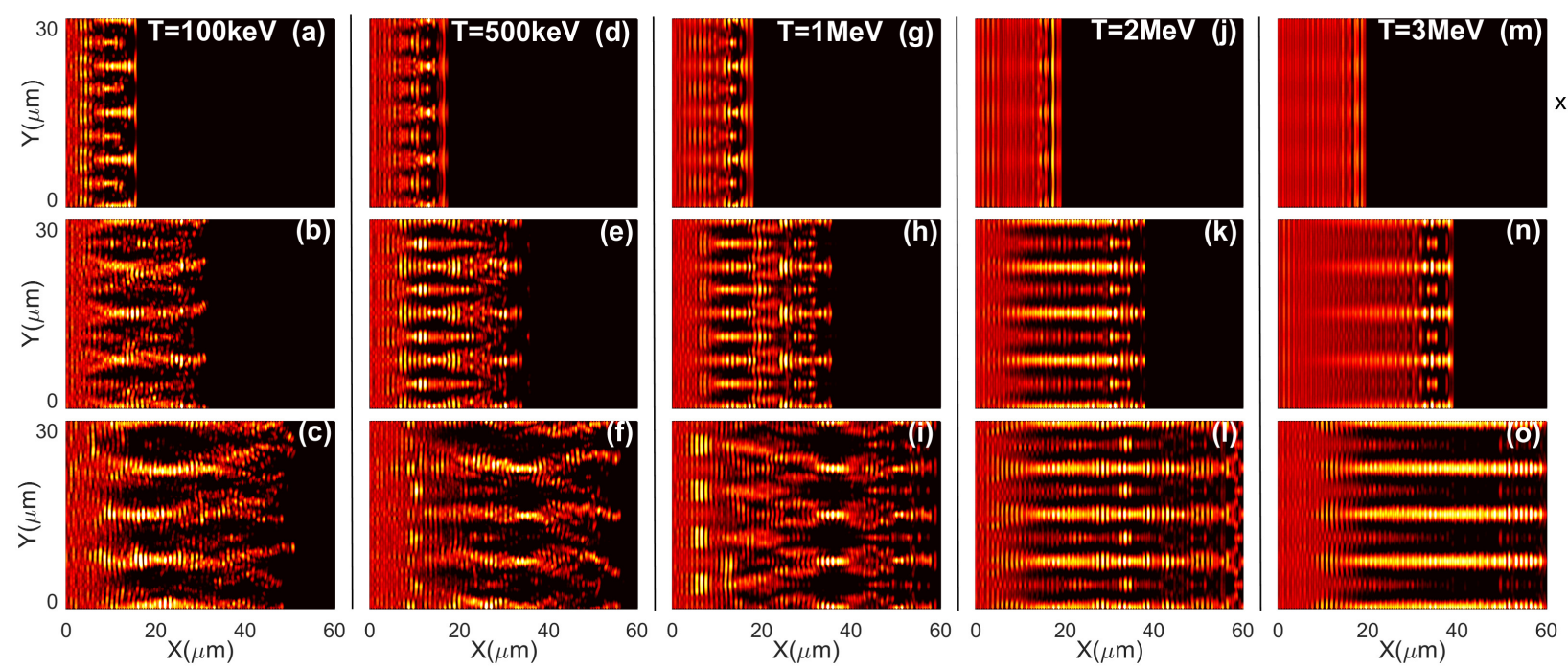

$\times 10^{12}(\mathrm{~V} / \mathrm{m})$
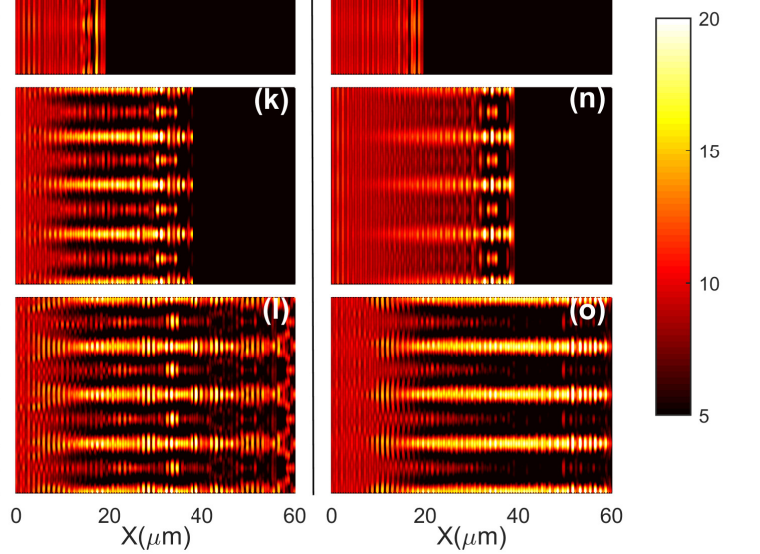

Fig. 1. Snapshots of the laser electric field strength inside the plasma. From left to right, the columns correspond, respectively, to plasma temperatures: $T=100 \mathrm{keV}, 500 \mathrm{keV}, 1 \mathrm{MeV}, 2 \mathrm{MeV}$ and $3 \mathrm{MeV}$. The first, 2nd and 3rd rows show snapshots at time $t=75 \mathrm{fs}, 150 \mathrm{fs}$ and $250 \mathrm{fs}$ respectively.

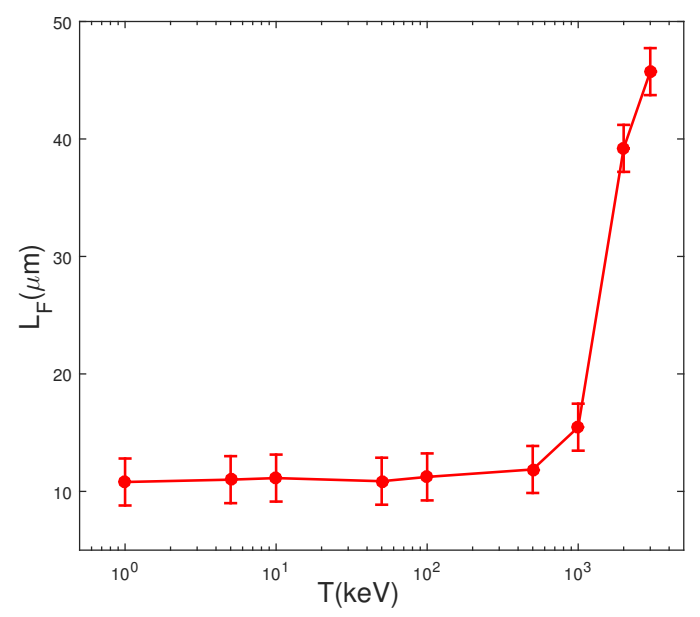

Fig. 2. Temperature dependence of the filamentation length, $L_{F}$. 


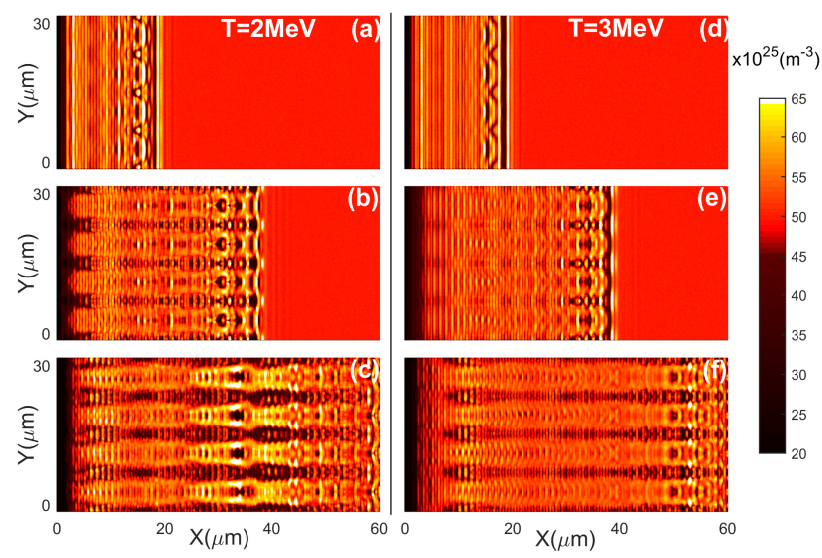

Fig. 3. Snapshots of the electron number density that correspond to the 4th and 5th columns of Fig. 1.

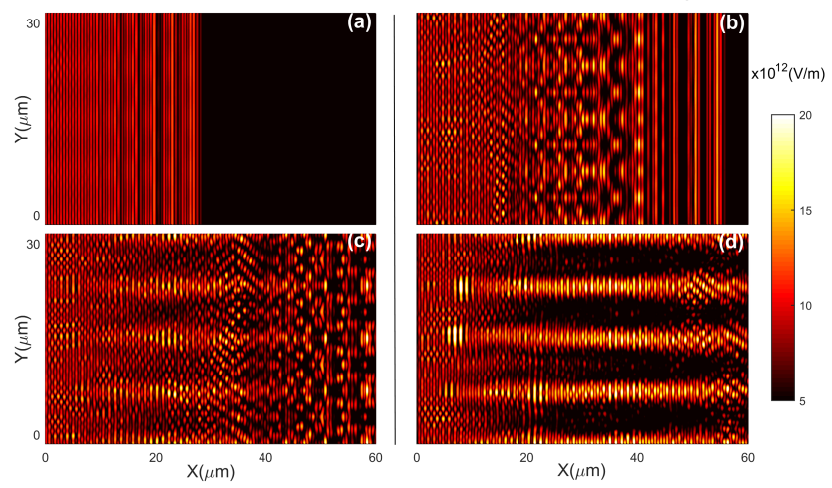

Fig. 4. Snapshots of the laser electric field strength inside a plasma with $T=100 \mathrm{keV}$ and $m^{*}=6 m$, taken at $t=100 \mathrm{fs}(\mathrm{a}), 200 \mathrm{fs}(\mathrm{b}), 300 \mathrm{fs}$ (c) and $400 \mathrm{fs}(\mathrm{d})$. 